\title{
CONCLUSIONS DES DISCUSSIONS SUR L'EVAPORATION
}

\section{L'évaporation est limitée par deux facteurs :}

a par la quantité d'eau susceptible d'être évaporée,

b par la quantité d'énergie disponible pour la vaporisation de l'eau.

2 Le cas extrême où la quantité d'énergie est élevée et l'eau disponible faible, introduit de grandes difficultés dans l'interprétation physique complète du phénomène; il soulève les problèmes de la circulation de l'eau en sols non saturés et celui de la réponse des plantes aux tensions élevées. Ces conditions caractérisent le problème agricole en zones arides.

3 A l'autre extrême où l'eau est en abondance, on peut appliquer avec succès des principes physiques connus. Ils conduisent aux principes généraux suivants : à un instant et en un lieu donnés, le taux d'évaporation sur une surface cultivée suffisamment vaste présente une limite supérieure qui ne peut être accrue par addition supplémentaire d'eau.

4 Le mécanisme physique de l'évaporation (l'eau n'étant pas limitante) fait intervenir des transferts et des transformations d'énergie, certains aspects de ces transferts étant contrôlés par les facteurs aérodynamiques.

5 L'analyse de ce mécanisme complexe montre que ces échanges d'énergie et ces transferts aérodynamiques sont météorologiques dans leur essence même, ce qui conduit à un autre principe général : le taux maximum d'évaporation (tel qưil est défini au paragraphe 3) est déterminé en premier lieu par le climat.

6 Sous certaines réserves ce taux maximum semble largement indépendant de l'espèce cultivée ou du type de sol sur lequel pousse la plante. Il est dès lors commode de désigner ce taux maximum par un nom spécial : la transpiration potentielle (ou évapotranspiration potentielle); celle-ci peut alors être définie de la manière suivante:

Le taux de transpiration potentielle est le taux d'évaporation au niveau d'une surface suffisamment vaste, couverte d'une culture verte de petite taille ${ }^{1}$ ), cette culture étant en phase de croissance active ${ }^{2}$ ), recouvrant totalement le sol, d'une hauteur uniforme et ne manquant pas d'eau.

7 Le concept de la transpiration potentielle est utile même dans l'évaluation qualitative de l'évaporation.

8 Il y a plusieurs façons d'essayer d'évaluer la transpiration potentielle. Toutes font usage des données météorologiques. Les plus satisfaisantes tiennent compte à la fois des aspects énergétiques et aérodynamiques du processus d'évaporation.

1) Dans l'état actuel de l'investigation et de la recherche, il n'est guère possible de donner une définition précise au terme "culture verte de petite taille", l'expression "de petite taille" s'applique en fait aux plantes communément cultivées en agriculture et en horticulture.

2) "En phase de croissance active" implique que la culture n'est pas ou n'a pas été récemment sujette à un arrêt de croissance tel que cela pourrait arriver à la suite d'une forte gelée nocturne ou après la coupe à ras d'une plante fourragère: 
9 Des incertitudes d'ordre physique dans l'estimation de la transpiration potentielle proviennent de deux sources:

a Une connaissance incomplète

1 des échanges d'énergies radiantes qui ont lieu à l'air libre et sous verre;

2 des processus de transfert turbulent dans l'atmosphère.

b Difficulté de mesurer les valeurs physiques en cause.

10 Des incertitudes d'ordre biologique proviennent de plusieurs sources. Elles tiennent à la morphologie de l'espèce, au stade phyisologique et à l'activité de la plante. Bien que certains des problèmes soient de nature manifestement physique, il est clair que la coopération du biologiste est essentielle si l'on veut tenter de résoudre les difficultés présentes.

11 En dépit des insuffisances énumérées aux paragraphes 9 et 10 , il est clair que l'évaporation en agriculture (eau non limitante) est un phénomène essentiellement physique. Dans beaucoup de problèmes pratiques, le taux de transpiration peut être regardé comme commandé par les données météorologiques et peut être exprimé quantitativement si l'on connaît les valeurs des éléments climatiques en cause.

12 Avec des instruments précis et une grande habileté pour les manipuler, il est possible après des calculs quelque peu compliqués, d'évaluer le taux d'évaporation présente. C'est là un travail d'une importance fondamentale dans le cadre de la physique météorologique et dont les résultats ne peuvent manquer de profiter à la science agronomique et en fin de compte à la pratique agricole.

13 A l'heure actuelle cependant, il est très difficile de transposer ces résultats, obtenus pour de courts intervalles de temps, à des périodes qui aient une signification en agriculture et en hydrologie.

En attendant, pour servir de guide dans les applications au champ, on doit adopter une méthode moins parfaite faisant appel à des mesures plus faciles à obtenir et à des calculs plus simples. Sur le plan de la pratique agricole en effet, il existe une vaste gamme de problèmes qui n'exigent qu'une réponse approximative.

14 De telles méthodes sont utiles mais ne sont guère faciles à contrôler en raison de la difficulté de préciser l'épaisseur de la lame d'eau évaporée dans les conditions du champ. Toutefois les observations à l'échelle culturale sont suffisamment nombreuses pour montrer que les estimations de l'évaporation ainsi obtenues peuvent être utilisées dans le problème théorique et pratique de l'irrigation, spécialement quand celle-ci est destinée à maintenir l'humidité à un taux toujours suffisant.

Pour estimer la transpiration potentielle, on doit disposer des données météorologiques indispensables et la précision de l'estimation sera limitée comme il est indiqué aux paragraphes 9 et 10. A l'heure actuelle, il est risqué d'utiliser les formules approchées existantes pour évaluer les pertes en eau correspondant à de courtes périodes.

15 Les calculs relatifs à l'évaporation de vastes surfaces d'eau libre semblent être d'une précision raisonnable.

16 Pour appliquer les calculs au bilan de l'eau dans les zones où les pluies sont insuffisantes, on doit faire des hypothèses sur les différences existant entre 
les pertes en eau, potentielle et réelle. Les résultats sont sujets à une très grande incertitude même dans les zones humides où cependant d'utiles résultats ont été obtenus.

17 Ces hypothèses ont été nécessaires par suite de l'insuffisance de nos connaissances sur le taux de transmission de l'eau à travers le sol et la plante. Elles le demeureront jusqu'à ce que de nouvelles recherches biologiques et physiques aient abouti. Les techniques actuelles de mesure de l'humidité du sol ne conviennent pas à ces recherches, ce qui limite sérieusement les possibilités de progresser dans l'immédiat. Il est urgent d'améliorer ces techniques de mesure.

18 Une étroite collaboration entre physiciens, hydrologistes, biologistes et agronomes est essentielle. Une telle collaboration devrait être internationale et effective à tous les stades de la recherche. Du point de vue instrumental par exemple, on peut souhaiter que les chercheurs des différents pays puissent se communiquer aussi bien leurs échecs que leurs réussites. Il est souvent regrettable d'avoir à attendre l'arrivée d'une publication; il faudrait faciliter les consultations personnelles de nature confidentielle aussi bien avant que pendant les expériences. Il peut en résulter une grande économie de temps et également d'argent, un jugement critique satisfaisant ne pouvant toujours être fait par référence à la littérature seule.

19 Bien que l'importance de l'eau sur l'économie humaine soit universellement reconnue, les efforts tentés dans ce domaine de recherches, en vue d'assurer le contrôle de l'eau, n'est pas en rapport avec cette importance.

Dans ce domaine de recherches, c'est au physicien qu'il appartient de découvrir les lois du mouvement de l'eau et les phénomènes qui en découlent.

20 Recherche et applications doivent progresser parallèlement étant donné qu'il n'est ni nécessaire ni désirable d'atteindre la perfection scientifique avant d’offrir une aide pratique. La double tâche du physicien est claire : améliorer ses propres connaissances et intensifier son aide là où elle est nécessaire. 